Irish Math. Soc. Bulletin

Number 88, Winter 2021, 39-45

ISSN 0791-5578

\title{
Ireland's Participation in the 62nd International Mathematical Olympiad
}

\author{
MARK FLANAGAN
}

\begin{abstract}
An account of the Irish team's participation in IMO 2021 in St Petersburg, including team selection and preparation, the problems set at the Olympiad, the performance of the contestants, and acknowledgement of sponsors.
\end{abstract}

The $62^{\text {nd }}$ International Mathematical Olympiad (IMO) took place in St. Petersburg, Russia, from 14-24 July 2021. A total of 619 students (64 of whom were girls) participated from 107 countries. As in 2020, due to the Covid pandemic, contestants were unable to travel to Russia in person, instead sitting invigilated examinations in their home country.

The Irish delegation consisted of six students (see Table 1) accompanied by Andrew Smith (Deputy Leader, UCD), Mark Flanagan (Team Leader, UCD), Anca Mustata (Observer, UCC) and Eugene Gath (Observer, UL).

\begin{tabular}{|l|l|c|}
\hline Name & School & Year \\
\hline Ellen Li & The Institute of Education, Dublin & $6^{\text {th }}$ \\
Evan Grealish & Calasanctius College, Oranmore, Co. Galway & $6^{\text {th }}$ \\
Adam Crowe & St Benildus College, Kilmacud, Dublin 14 & $6^{\text {th }}$ \\
James Chen & Castletroy College, Newtown, Limerick & $4^{\text {th }}$ \\
Fionn Kimber O'Shea & Coliste an Phiarsaigh, Glanmire, Co. Cork & $3^{\text {rd }}$ \\
Taiga Murray & St Benildus College, Kilmacud, Dublin 14 & $5^{\text {th }}$ \\
\hline
\end{tabular}

TABLE 1 . The Irish contestants at the $62^{\text {nd }}$ IMO

\section{TEAM SELECTION AND PREPARATION}

The team detailed in Table 1 consisted of those six students (in order) who scored highest in the Irish Mathematical Olympiad (IrMO), which was held for the $34^{\text {th }}$ time on Saturday, $8^{\text {th }}$ May, 2021. The IrMO contest consists of two 3-hour papers on one day with five problems on each paper. In 2021, due to Covid restrictions, students sat the exam at home, under supervision of a trusted adult, usually a parent.

During the spring, students who participate in the IrMO usually attend extracurricular Mathematics Enrichment classes, which are offered at six Mathematics Enrichment Centres (UCC, UCD, NUIG, UL, MU and IT Sligo). These classes run each year from January until April and are offered by volunteer academic mathematicians from these universities or nearby third-level institutions. More information on the organisation of these classes, as well as links to the individual maths enrichment centres, can be found at the Irish Maths Enrichment / IrMO website, http://www.irmo.ie/.

2020 Mathematics Subject Classification. 00A99.

Key words and phrases. Mathematical Olympiad.

Received on 1-9-2021.

DOI:10.33232/BIMS.0088.39.45. 
However, in 2021 these in-person maths enrichment activities were not possible due to the Covid pandemic. Instead, in 2021 a collaborative maths enrichment / IMO training programme (involving all six centres mentioned above) was made available online; trainers developed video-based lecture materials and associated problem sets, and this material was posted online on a password-accessible webpage on the website of the Irish Mathematical Trust (IMT). This training included an entire course on olympiad problem-solving in geometry, organised and delivered by Anca Mustata (UCC).

The selection and training for IMO 2021 followed procedures which are by now wellestablished. First, an Irish Maths Olympiad "Squad" was identified, consisting of the top performers in IrMO 2020 who were eligible to qualify for the Irish IMO team in 2021. For these students, various training activities were organised. First, the members of the Irish IMO Squad were directed to engage with the online training materials mentioned in the previous paragraph, and for each online training session the students could return their solutions or attempts to the homework problems by email to the proposer of the problems before a given deadline. The problem proposer then provided feedback on their work, as well as either full solutions or else hints on how to make further progress. This type of training is important for the successful engagement of "returning" students, and helps to develop the students' independence in mathematical problem solving.

Other training activities usually take the form of training camps, where students would travel to a central location in Ireland to engage in problem-solving activities. Such training activities are important, as during these mathematically intense $3-5$ day events, students have the opportunity to socialise with their peers, exchange their mathematical ideas, and increase their motivation for their work throughout the year. However, due to Covid-related travel restrictions as well as restrictions on indoor activities, this year the training activities were exclusively in an online and remote format.

At the beginning of June, each of the team members was assigned a personal mentor whose role was to help the team member to structure their own personal training programme during the weeks prior to the IMO. This allowed each individual team member to tailor their efforts on each problem-solving area according to their relative strength or weakness in that particular area.

A final online team training camp took place from 12-16 July 2021, consisting of two four-hour sessions on each day. Each session provided a mix of presentation of new problem-solving materials and techniques, as well as independent problem-solving activities for the students. Typically each session would end with the students presenting and discussing their own solutions to the assigned problems.

\section{Jury, Problem Selection and Examinations}

In normal years, a "jury" consisting of national team leaders meets to select the IMO contest problems from a shortlist developed by the host country. Team leaders are then sequestered in a location sufficiently distant from that of the team and forbidden from communicating with the team or Deputy Leader until the end of the second day of examinations.

In 2021, as in 2020, such in-person jury meetings were not possible, and the problems were selected entirely by the problem selection committee, which was appointed by the host country. While this deprived national team leaders of the opportunity to contribute to problem selection, it also allowed us to relax the communication embargo, so that team leaders could continue to be involved in the logistics of organising local contests.

Unlike in 2020, in 2021 Covid-related travel restrictions were sufficiently relaxed in Ireland to allow all team members to travel to a central location to take the IMO exams. The IMO exams were held in the Clayton Hotel in Ballsbridge, Merrion Road, 
Dublin. For the two contest days, the IMO organisers required the appointment of an independent commissioner who was not involved in the team training or selection processes, a resident but not a citizen of the competing country. The responsibilities of the commissioner included receiving the examination question papers, invigilating the examination and scanning scripts for transmission to Russia at the end of each examination. In Ireland, the IMO Commissioner was Jon-Ivar Skullerud, who also served as IMO Commissioner in 2020.

The final marks for each contestant are agreed in a process known as coordination. This important part of the IMO is well-established and ensures that the scripts of the students from so many different nations are marked fairly and consistently. The decisions in this process are based on detailed and strict marking schemes prepared by the coordination teams.

The marking of the scripts of each participating country is undertaken by two independent groups. One group consists of the Team Leader, the Deputy Leader and Official Observers. The second group consists of the coordinators, who are appointed by the local organisers. In contrast to the usual IMO procedures, in 2021 the coordination process was undertaken entirely online, but the online process represented the usual face-to-face coordination meetings very faithfully.

\section{THE PROBLEMS}

The two exams took place on the $19^{\text {th }}$ and $20^{\text {th }}$ of July, with each exam having to start within a prescribed time window so that no student anywhere in the world would start an IMO exam after another student somewhere else had finished it. In Ireland, the starting time for each exam was $10 \mathrm{am}$. On each day, $4 \frac{1}{2}$ hours were available to solve three problems. The problems are published at http://www.imo-official.org/problems.aspx after each examination.

\section{FIRST DAY}

Problem 1. Let $n \geqslant 100$ be an integer. Ivan writes the numbers $n, n+1, \ldots, 2 n$ each on different cards. He then shuffles these $n+1$ cards, and divides them into two piles. Prove that at least one of the piles contains two cards such that the sum of their numbers is a perfect square.

Problem 2. Show that the inequality

(Australia)

$$
\sum_{i=1}^{n} \sum_{j=1}^{n} \sqrt{\left|x_{i}-x_{j}\right|} \leqslant \sum_{i=1}^{n} \sum_{j=1}^{n} \sqrt{\left|x_{i}+x_{j}\right|}
$$

holds for all real numbers $x_{1}, \ldots, x_{n}$.

(Canada)

Problem 3. Let $D$ be an interior point of the acute triangle $A B C$ with $A B>A C$ so that $\angle D A B=\angle C A D$. The point $E$ on the segment $A C$ satisfies $\angle A D E=\angle B C D$, the point $F$ on the segment $A B$ satisfies $\angle F D A=\angle D B C$, and the point $X$ on the line $A C$ satisfies $C X=B X$. Let $O_{1}$ and $O_{2}$ be the circumcentres of the triangles $A D C$ and $E X D$, respectively. Prove that the lines $B C, E F$, and $O_{1} O_{2}$ are concurrent.

(Ukraine) 


\section{SECOND DAY}

Problem 4. Let $\Gamma$ be a circle with centre $I$, and $A B C D$ a convex quadrilateral such that each of the segments $A B, B C, C D$ and $D A$ is tangent to $\Gamma$. Let $\Omega$ be the circumcircle of the triangle $A I C$. The extension of $B A$ beyond $A$ meets $\Omega$ at $X$, and the extension of $B C$ beyond $C$ meets $\Omega$ at $Z$. The extensions of $A D$ and $C D$ beyond $D$ meet $\Omega$ at $Y$ and $T$, respectively. Prove that

$$
A D+D T+T X+X A=C D+D Y+Y Z+Z C .
$$

(Poland)

Problem 5. Two squirrels, Bushy and Jumpy, have collected 2021 walnuts for the winter. Jumpy numbers the walnuts from 1 through 2021, and digs 2021 little holes in a circular pattern in the ground around their favourite tree. The next morning Jumpy notices that Bushy had placed one walnut into each hole, but had paid no attention to the numbering. Unhappy, Jumpy decides to reorder the walnuts by performing a sequence of 2021 moves. In the $k$-th move, Jumpy swaps the positions of the two walnuts adjacent to walnut $k$.

Prove that there exists a value of $k$ such that, on the $k$-th move, Jumpy swaps some walnuts $a$ and $b$ such that $a<k<b$.

(Spain)

Problem 6. Let $m \geqslant 2$ be an integer, $A$ be a finite set of (not necessarily positive) integers, and $B_{1}, B_{2}, B_{3}, \ldots, B_{m}$ be subsets of $A$. Assume that for each $k=1,2, \ldots, m$ the sum of the elements of $B_{k}$ is $m^{k}$. Prove that $A$ contains at least $m / 2$ elements.

(Austria)

\section{The Results}

The contest problems are chosen such that Problems 1 and 4 are the most accessible, while Problems 2 and 5 are more challenging. Problems 3 and 6 are usually the most difficult problems, whose existence on the paper is justified in posing a sizeable challenge even to the top students in the IMO competition. Table 2, which shows the scores achieved by all contestants on the 6 problems, illustrates that this gradient of difficulty was generally maintained this year also. However, Problem 2 was significantly more difficult than usual, its difficulty level being more aligned with Problems 3 and 6. From the table it can also be seen that for many problems, the marks were either close to 0 or close to 7 , with partial marks difficult to obtain. This is a reflection of the marking schemes for the problems, which were somewhat unforgiving this year. These are indicators that this was a particularly difficult IMO contest.

In fact, from the available statistical information it can be concluded that IMO 2021 was the most difficult IMO in which an Irish team has ever participated. This may be seen by considering the average percentage score in the IMO contest, calculated as the total number of points scored altogether by the 619 participants (i.e., 7175 ) divided by the maximum possible total score (i.e., $42 \times 619$ ). This year, this was $27.6 \%$. This lies in stark contrast to the average percentage scores for the past 5 years (2016-2020), which were $35.2 \%, 34.7 \%, 36.8 \%, 37.8 \%$ and $37.0 \%$ respectively. In fact, this is the lowest average percentage score in any IMO since Ireland's first participation in 1988.

Table 3 shows the results of the Irish contestants. One student (Taiga Murray) was awarded an Honourable Mention for his perfect solution to Problem 5 - at such a difficult IMO, this is a fantastic achievement. However, the scores are generally lower than in recent years - this is partly due to the difficulty of this year's contest (and in particular the marking schemes for the problems), but may also be due to the effect of the Covid 


\begin{tabular}{|c||r|r|r|r|r|r|}
\hline & P1 & P2 & P3 & P4 & P5 & P6 \\
\hline 0 & 131 & 522 & 488 & 218 & 404 & 562 \\
1 & 36 & 61 & 110 & 33 & 12 & 12 \\
2 & 41 & 12 & 4 & 39 & 13 & 2 \\
3 & 10 & 2 & 1 & 2 & 4 & 3 \\
4 & 41 & 3 & 1 & 12 & 2 & 1 \\
5 & 39 & 1 & 0 & 1 & 5 & 2 \\
6 & 35 & 2 & 0 & 5 & 4 & 0 \\
7 & 286 & 16 & 15 & 309 & 175 & 37 \\
\hline average & 4.393 & 0.375 & 0.372 & 3.817 & 2.152 & 0.481 \\
\hline
\end{tabular}

TABLE 2. The number of contestants achieving each possible number of points on Problems 1-6

\begin{tabular}{|l|r|r|r|r|r|r||c|c|}
\hline Name & P1 & P2 & P3 & P4 & P5 & P6 & total & ranking \\
\hline Taiga Murray & 0 & 0 & 0 & 1 & 7 & 0 & 8 & 348 \\
James Chen & 2 & 0 & 0 & 0 & 0 & 0 & 2 & 470 \\
Ellen Li & 1 & 0 & 0 & 0 & 0 & 0 & 1 & 499 \\
Evan Grealish & 1 & 0 & 0 & 0 & 0 & 0 & 1 & 499 \\
Adam Crowe & 0 & 0 & 0 & 0 & 0 & 0 & 0 & 533 \\
Fionn Kimber O'Shea & 0 & 0 & 0 & 0 & 0 & 0 & 0 & 533 \\
\hline
\end{tabular}

TABLE 3. The results of the Irish contestants

restrictions on our national Mathematics Enrichment programme over the last two years, which has no doubt impacted on the students' ability to train for this challenging contest. Having said this, the Irish contestants had some good ideas on some of the problems which were unfortunately not rewarded by the marking schemes. For example, for Problem 4, all of the points in the official marking scheme hinged on spotting and proving an initial symmetry $(I X=I Y, I T=I Z, X T=Y Z, X Z=Y T)$ that was difficult to spot. However, while not necessarily rewarded by the marking scheme, the Irish contestants had good ideas on this problem, such as using the tangency points with the incircle of the quadrilateral $A B C D$ to simplify the required relation, finding similar triangles and ratios involving the segments in the required relation, and recognising the relevance of the incentre-excentre lemma to this problem. Also, for Problem 1 James Chen managed to solve the case $n=100$ by showing the existence of a 5 -cycle in the graph representing the problem, the difficulty of which task was appreciated by the Russian coordinators.

The figures in Table 4 have the following meaning. The first figure after the topic indicates the percentage of all points scored out of the maximum possible. The second number is the same for the Irish team and the final column indicates the Irish average score as a percentage of the overall average.

\section{OUTLOOK}

The next countries to host the IMO will be

2022 Norway

2023 Japan

2024 Ukraine

2025 Australia 


\begin{tabular}{|c|r|r|r|r|}
\hline Problem & topic & all countries & Ireland & relative \\
\hline 1 & number theory & 62.8 & 9.5 & 15.2 \\
2 & algebra & 5.4 & 0.0 & 0.0 \\
3 & geometry & 5.3 & 0.0 & 0.0 \\
4 & geometry & 54.5 & 2.4 & 4.4 \\
5 & combinatorics & 30.7 & 16.7 & 54.2 \\
6 & algebra & 6.9 & 0.0 & 0.0 \\
\hline all & & 27.6 & 4.8 & 17.3 \\
\hline
\end{tabular}

TABLE 4. Relative results of the Irish team for each problem

\section{Conclusions}

The difficulty of the problems at IMO 2021, as well as the corresponding marking schemes, made it difficult this year for students to solve the contest problems as well as to obtain partial credit for their ideas. This level of difficulty is indicative that there is a need for the IMO Jury to be reinstated in the problem selection process. Despite this, one Irish student obtained an Honorable Mention, which is a great achievement considering the difficulty of the contest as well as a lack of face-to-face training in problemsolving. It is hoped that regular face-to-face maths enrichment and IMO training will again become possible in Ireland in the not-too-distant future. This training, as well as Ireland's continued participation in these contests, require sustained funding.

\section{ACKNOWLEDGEMENTS}

Ireland could not participate in the International Mathematical Olympiad without the continued financial support of the Department of Education, which is gratefully acknowledged. Thanks to the Minister of Education, Norma Foley TD, and the members of her department, especially Aoibhín O'Malley, for their continuing help and support. Also, thanks to the Royal Irish Academy, its officers, its Physical, Chemical and Mathematical Sciences Committee, and especially Teresa Gallagher, for support in obtaining funding.

The principal foundation for the success of the contestants is the work done with the students in the Mathematics Enrichment Programmes at six third level institutions. This work is carried out for free by volunteers in their spare time. Many thanks to all those involved in the Mathematics Enrichment activities and training camps, which were all held virtually in 2021. The remote Mathematics Enrichment activities were delivered in 2021 by Kazim Buyukboduk, Mark Flanagan, Mark Fortune, Eugene Gath, Mayya Golitsyna, Jonathan Grant Peters, David Malone, Myrto Manolaki, John Murray, Seoirse Murray, Anca Mustata, Andrei Mustata, Andrew Smith, Rob Sparkes, Maria Tieder and Jessica Weitbrecht. The organisation of these sessions and the collation of all video materials, problem sets, etc., as well as their presentation on the IMT website, was managed by Anca Mustata. At the IMO team training camp, which was organised by Mark Flanagan, the trainers were Mark Flanagan, Eugene Gath, Bernd Kreussler, Gordon Lessells, Myrto Manolaki, Anca Mustata and Andrew Smith. The trainers who served as personal mentors to the IMO team members during the weeks prior to the IMO contest were Mark Flanagan, Mayya Golitsyna, Bernd Kreussler, Gordon Lessells, Seoirse Murray and Anca Mustata. Thanks also to UCC, UCD, NUIG, UL, MU and IT Sligo for permitting the use of their facilities in the delivery of the national Maths Enrichment Programme.

Thanks to the staff at the Clayton Hotel, Ballsbridge, Merrion Road, Dublin, for their excellent hospitality and for helping us to ensure that everything went smoothly 
during the contest days at IMO 2021. Finally, thanks to the hosts for organising this year's IMO virtually from Russia, and thanks to the Russian guides Leyla Kanbarova and Anastasiya Shurpitskaya, who helped to make the Irish team members' experience at IMO 2021 a truly international one.

Mark Flanagan is an Associate Professor in Electronic Engineering at University College Dublin. Since 2010 he has been actively involved in delivering Mathematics Enrichment classes and in training talented second-level students for the International Mathematical Olympiad. He is serving as Treasurer of the Irish Mathematical Trust since 2013.

School of Electrical and Electronic Engineering, University College Dublin, Belfield, DUBLIN 4, IRELAND.

E-mail address: mark.flanagan@ucd.ie 